\title{
Long-term outcomes of per-oral endoscopic myotomy in achalasia patients with a minimum follow-up of 4 years: a multicenter study
}

\section{다(1) $(2)$}

\section{Authors}

Olaya I. Brewer Gutierrez ${ }^{1}$, Robert A. Moran², Pietro Familiari ${ }^{3}$, Mohamad H. Dbouk ${ }^{1}$, Guido Costamagna ${ }^{3}$, Yervant Ichkhanian ${ }^{1}$, Stefan Seewald ${ }^{4}$, Amol Bapaye ${ }^{5}$, Joo Young Cho ${ }^{6}$, Maximilien Barret ${ }^{7}$, Nikolas Eleftheriadis ${ }^{8}$, Mathieu Pioche $^{9}$, Bu' Hussain Hayee ${ }^{10}$, Marcel Tantau ${ }^{11}$, Michael Ujiki ${ }^{12}$, Rosario Landi ${ }^{3}$, Martina Invernizzi ${ }^{4}$, In Kyung Yoo ${ }^{6}$, Sabine Roman ${ }^{9}$, Amyn Haji ${ }^{10}$, H. Mason Hedberg ${ }^{12}$, Nasim Parsa ${ }^{1}$, Francois Mion ${ }^{9}$, Lea Fayad ${ }^{1}$, Vivek Kumbhari ${ }^{1}$, Anant Agarwalla ${ }^{1}$, Saowanee Ngamruengphong ${ }^{1}$, Omid Sanaei ${ }^{1}$, Thierry Ponchon ${ }^{9}$, Mouen A. Khashab ${ }^{1}$

Institutions

1 Division of gastroenterology and hepatology Johns Hopkins Hospital, Baltimore, United States

2 Department of Gastroenterology and Hepatology, Medical University of South Carolina, Charleston, South Carolina, United States

3 Digestive Endoscopy Unit - Gemelli University Hospital, Rome, Italy

4 Center of Gastroenterolgy Hirslanden Private Clinic Group Witellikerstrasse 40, 8032 Zürich

5 Shivanand Desai Centre for Digestive Disorders, Deenanath Mangeshkar Hospital and Research Centre, Pune, India

6 Digestive Endoscopy Center, CHA Bundang Hospital, South Korea

7 Department of gastroenterology, Cochin Hospital Paris, France

8 Metropolitan Hospital, Athens, Greece

9 Gastroenterology unit/unit of functional disease and digestive physiology, Edouard Herriot hospital, Lion, France

10 Institute of Therapeutic Endoscopy, King's College Hospital NHS Foundation Trust, London, UK

11 Iuliu Hațieganu University of Medicine and Pharmacy, Cluj-Napoca, Romania

12 NorthShore University HealthSystem/University of Chicago Medical Center, Chicago, United States

submitted 25.4.2019

accepted after revision $\quad 27.1 .2020$

Bibliography

DOI https://doi.org/10.1055/a-1120-8125 |

Endoscopy International Open 2020; 08: E650-E655

(c) Georg Thieme Verlag KG Stuttgart · New York elSSN 2196-9736
Corresponding author

Mouen A. Khashab, MD, Johns Hopkins Hospital, 1800

Orleans Street, Sheikh Zayed Tower, Baltimore, MD 21287

Fax: +1- 410-502-7010

mkhasha1@jhmi.edu

\section{ABSTRACT}

Background and study aims Per-oral endoscopic myotomy (POEM) is associated with a short-term clinical response of $82 \%$ to $100 \%$ in treatment of patients with achalasia. Data are limited on the long-term durability of the clinical response in these patients. The aim of this study was to determine the long-term outcomes of patients undergoing POEM for management of achalasia.

Methods This was a retrospective multicenter cohort study of consecutive patients who underwent POEM for management of achalasia. Patients had a minimum of 4 years follow-up. Clinical response was defined by an Eckardt score $\leq 3$.

Results A total of 146 patients were included from 11 academic medical centers. Mean $( \pm$ SD) age was $49.8( \pm 16)$ years and 79 (54\%) were female. The most common type of achalasia was type II, seen in $70(47.9 \%)$ patients, followed by type I seen in 41 (28.1\%) patients. Prior treatments included: pneumatic dilation in 29 (19.9\%), botulinum toxin injection in 13 (8.9\%) and Heller myotomy in seven patients ( $4.8 \%$ ). Eight adverse events occurred ( 6 mucosotomies, 2 pneumothorax) in eight patients (5.5\%). Median follow-up duration was 55 months (IQR 49.9-60.6). Clinical response was observed in 139 (95.2\%) patients at follow-up of $\geq 48$ months. Symptomatic reflux after POEM was seen in 45 (32.1\%) patients, while $35.3 \%$ of patients were using daily PPI at 48 months post POEM. Reflux esophagitis was noted in $16.8 \%$ of patients who underwent endoscopy.

Conclusion POEM is a durable and safe procedure with an acceptably low adverse event rate and an excellent long- 


\section{Introduction}

Per-oral endoscopic myotomy (POEM) is a minimally invasive endoscopic technique for the treatment of achalasia and spastic esophageal disorders. It has been increasingly adopted worldwide with more than 7000 procedures performed to date $[1,2]$.

In comparison to laparoscopic Heller myotomy, POEM is as effective in short-term follow-up, with a favorable safety profile and a less invasive nature [3-8]. Short-term clinical response has been reported anywhere between $82 \%$ to $100 \%$ [5, 9-11]. There is scarce long-term data available. A single-center study from Japan demonstrated a durable clinical response that persisted for up to 3 years [5]. A single-center clinical study from China demonstrated that the long-term clinical success after POEM decreased slightly over time [12], with clinical success dropping from $94 \%$ at 1 year to $87 \%$ at 5 years. Recently, Teiltembaum et al reported a single-center experience 5 years after POEM in 36 patients; $83 \%$ of patients with achalasia had symptomatic success (Eckardt $\leq 3$ ) at 6 months and maintained at 2 years; however, there was a small but significant worsening of symptoms between 2 and 5 years [13]. Available data compare favorably to laparoscopic Heller myotomy (LHM) with clinical success reported to be $76.1 \%$ at 5 years [14].

The primary aim of the current study was to evaluate the long-term clinical response in patients undergoing POEM for the management of achalasia in a multicenter cohort. Secondary aims were to evaluate the incidence of reflux and determination of factors associated with long-term clinical failure.

\section{Methods}

This was a retrospective, international, multicenter cohort study including 11 tertiary centers ( 2 from the United States, 7 from Europe, 2 from Asia). The total number of patients contributed by each center were as follows: Gemelli University Hospital, Italy (101), Hirslanden Private Clinic Group, Zürich (19), Deenanath Mangeshkar Hospital, India (18), Johns Hopkins Hospital, US (17), Edouard Herriot hospital, France (13), CHA Bundang Hospital, South Korea (7), Cochin Hospital, France (6), Metropolitan Hospital, Greece (5), King's College Hospital, UK (3), Iuliu Hațieganu University of Medicine and Pharmacy, Romania (2), NorthShore University HealthSystem, United States (2). Institutional Review Board approval was obtained at all institutions for chart review and patient follow-up. We looked at all consecutive adult patients ( $>18$ years) who underwent POEM for management of any achalasia subtype (type I, II and III) between May 2011 and November 2013. Patients were excluded if they underwent POEM for an esophageal motility disorder other than achalasia. The approach to data collection differed according to the center. For most centers, the researchers attempted to get the latest follow-up on their patients. For those that we were able to get a follow-up on, at or beyond the 48-month end point, we did a full chart review to collect clinical data. Some centers had their own protocols in place to collect data prospectively on all patients that underwent POEM. From our cohort, 11 patients were included in a prior study by our group about gastroesophageal reflux disease (GER) after POEM [15]; similarly, all cases shared from the Italian center were also included in another study [7]. However, long-term data beyond 2 years have not been reported previously on these patients.

In addition to baseline demographic and clinical data, we collected: details of preoperative high-resolution esophageal manometry (HREM), type of Achalasia, prior therapies, technical details during POEM, adverse events (AEs), post-POEM reflux symptoms, presence of esophagitis on follow-up upper endoscopy (EGD), post-procedure $\mathrm{pH}$ testing, proton pump inhibitor (PPI) use, HREM post-POEM and additional treatment of patients who failed POEM.

POEM technique has been previously described in multiple studies [1]. The procedure involves the use of a per-oral cap-fitted endoscopic approach under general anesthesia and with positive pressure ventilation. The standard technique following the principles of POEM was used at all involved institutions. The four sequential steps were universally performed, mucosal incision at the 1-2 o'clock position of the esophageal wall (anterior approach) or at the 5-6 o'clock position (posterior approach) was done, followed by the creation of a submucosal tunnel using the ESD knife of choice and swift or spray coagulation mode at $50 \mathrm{~W}$, effect 2 . At the discretion of the endoscopist, either selective inner circular or full-thickness myotomy was performed using a triangular tip, hook or hybrid knife and spray coagulation current at $50 \mathrm{~W}$, effect 2 . Following the completion of the myotomy, the mucosal entry was closed using endoscopic clips. The post-procedure protocol may have also varied between centers. In general, patients were admitted overnight for IV hydration and pain management. Patients were kept NPO overnight and on postoperative day one, an esophagram with water soluble contrast was performed to rule out extraesophageal leakage of contrast. Patients were then started on a soft diet and discharged home. Patients follow-up was performed either in an outpatient clinic or by a phone interview. Follow-up intervals were between 1 and 6 months post-POEM and thereafter at 12, 24, 36 and 48 months post-procedure.

\section{Outcomes and definitions}

An Eckardt score $\leq 3$ post-POEM defined clinical response [16]. In addition to the Eckardt score at 48 months, Eckardt scores when available were recorded at 6, 12, 24 and 36 months post-POEM. Adverse events (AEs) were defined as symptomatic or procedural/post-procedural events that required any type of additional intervention or prolongation of hospital stay. AE severity was graded according to the ASGE lexicon classification for endoscopic AEs [17]. Post-POEM gastroesophageal reflux was defined as: 1) typical symptoms consistent with heartburn and or regurgitation reported by the patient during post-POEM follow-up obtained by chart review/telephone interview; 2) presence of esophagitis ( graded according to the LA classification) on follow-up endoscopy; and/or 3) a DeMeester score $>14.72$ on $\mathrm{pH}$ monitoring and abnormal acid exposure time. Abnormal acid exposure time was defined as $\mathrm{pH}<4$ more than $6 \%$ of the time, off-PPI. 


\section{Statistical analysis}

Descriptive statistics were presented as frequencies (\%) for categorical variables, mean \pm standard deviation (SD) for continuous variables or median (interquartile range [IQR]) for ordinal or other continuous variables. Chi-square test and Fisher's exact test were used to compare categorical data while Student's t-test, Wilcoxon signed ranks-andmedian tests were used for continuous data and ordinal data, as appropriate. $P \leq 0.05$ was considered statistically significant. Statistical analysis was performed using SPSS software (SPSS version 25, Chicago, Illinois, United States).

\section{Results}

POEM was performed in 193 patients with Achalasia during the specified study period; 47 patients were excluded because they didn't have follow-up at or after 48 months. Of the remaining 146 patients, 79 (54\%) were females, $110(75.3 \%)$ were white, mean $( \pm S D)$ age of $49.8( \pm 16)$ years. Achalasia subtypes included $41(28.1 \%)$ type I, $70(47.9 \%)$ type II, 9 (6.2\%) type III and $26(17.8 \%)$ were not specified type. Forty-one (41) patients (28.1\%) had one or more prior therapies: 13 patients (8.9\%) underwent botulinum toxin injection, 29 (19.9\%) had pneumatic dilation, and 7 (4.8\%) had a prior LHM. Data on preoperative LES resting pressure and integrated relaxation pressure (IRP) on HREM was available in 93 of 146 patients. Mean ( \pm SD) resting pressure was $20.8( \pm 8.4) \mathrm{mmHg}$ whereas mean $( \pm S D)$ IRP was $26.9( \pm 13.1) \mathrm{mmHg}$. Median (IQR) preoperative Eckardt score was 7 (6.75-9) ( $\bullet$ Table 1$)$.

Regarding technical details during POEM, the majority of patients had an anterior approach $(n=112,77.8 \%)$. The mean $( \pm \mathrm{SD})$ length of the esophageal and gastric myotomies was $8.5 \mathrm{~cm}( \pm 2.5)$ and $3.2 \mathrm{~cm}( \pm 1)$, respectively. More than half of the cohort had a full-thickness myotomy at the level of the lower esophageal sphincter $(n=76 / 145,52.4 \%)(\triangleright$ Table 1$)$.

\section{Outcomes}

Clinical success was achieved in $96.4 \%, 95.9 \%, 94.1 \%, 95.9 \%$, and $95.2 \%$ of patients at $6,12,24,36$ and $\geq 48$ months after POEM, respectively ( $\triangleright$ Fig. $1, \triangleright$ Table 2 ). There was no statistically significant difference in clinical response at $\geq 48$ months post-POEM based on the achalasia subtypes (Type $I=97.6 \%$, Type $I I=94.3 \%$, Type $I I I=88.9 \%$, Unspecified $=96.2 \% ; P=$ 0.690). Patients were followed-up for a median (IQR) of 55 months (49.9-60.6) post-POEM.

Median (IQR) Eckardt score decreased from 7 (6.75-9) at baseline to $1(0-2)(P<0.001)$ at 48 months post-POEM. Similarly, there was no difference in the Eckardt score at 48 months post-POEM based on the achalasia subtypes (Type I: $1(0.5-2)$, Type II: 1 (0-2), Type III: 1 (0-2.5), Unspecified: $1(0-2)$; $P=$ $0.267)$. There was a significant decrease in the LES resting pressure from $38.4( \pm 20.8)$ to $16.9( \pm 9) \mathrm{mmHg}(P<0.001)$ and the mean IRP from $26.9( \pm 13.1)$ to $10.28( \pm 7.1)(P<0.001)$, respectively.

Regarding the seven patients who experienced clinical failure at 48 months, five experienced initial response (within 6
- Table 1 Baseline demographic and pre-POEM characteristics.

\begin{tabular}{|c|c|c|}
\hline & $\begin{array}{l}\text { Number of pa- } \\
\text { tients with avail- } \\
\text { able data } N=146 \\
\text { if not specified }\end{array}$ & $\begin{array}{l}\text { Overall } \\
\mathrm{N}(\%)\end{array}$ \\
\hline Age; $($ mean \pm SD) & & $49.8 \pm 16$ \\
\hline BMI; (mean \pm SD) & 108 & $23 \pm 4.7$ \\
\hline Female; (n) (\%) & & $79(54)$ \\
\hline \multicolumn{3}{|l|}{ Ethnicity; (n) (\%) } \\
\hline - White & & $110(75.3)$ \\
\hline - Black & & $8(5.5)$ \\
\hline - Asian & & $26(17.8)$ \\
\hline - Others & & $2(1.4)$ \\
\hline \multicolumn{3}{|l|}{ Disease Classification; (n) (\%) } \\
\hline - Typel & & $41(28.1)$ \\
\hline - Type II & & 70 (47.9) \\
\hline - Type III & & $9(6.2)$ \\
\hline - Unspecified Achalasia & & $26(17.8)$ \\
\hline Prior therapy (yes); (n) (\%) & & $41(28.1)$ \\
\hline Pneumatic dilation; (n) (\%) & & $29(19.9)$ \\
\hline Botox; (n) (\%) & & $13(8.9)$ \\
\hline Heller myotomy; (n) (\%) & & $7(4.8)$ \\
\hline $\begin{array}{l}\text { Sigmoidal esophageal } \\
\text { shape; (n) (\%) }\end{array}$ & 142 & $10(7)$ \\
\hline $\begin{array}{l}\mathrm{HRM} I R P(\mathrm{mmHg}) \\
(\text { mean } \pm \mathrm{SD})\end{array}$ & 93 & $26.9 \pm 13.1$ \\
\hline $\begin{array}{l}\text { HRM resting pressure } \\
(\mathrm{mmHg}) ;(\text { mean } \pm \mathrm{SD})\end{array}$ & 125 & $38.4 \pm 20.8$ \\
\hline $\begin{array}{l}\text { Pre-POEM Eckardt score; } \\
\text { (median) (IQR) }\end{array}$ & & $7(6.75-9)$ \\
\hline \multicolumn{3}{|c|}{$\begin{array}{l}\text { HREM, high-resolution esophageal manometry; IRP, integrated relaxation } \\
\text { pressure; LES, lower esophageal sphincter; IQR, interquartile range; PPI, } \\
\text { proton pump inhibitor }\end{array}$} \\
\hline
\end{tabular}

months), having late recurrence of symptoms. Of these, four patients underwent retreatment while the other three opted not to undergo further therapy: one had a repeated POEM and three had pneumatic balloon dilation. Clinical response was attained in three of four patients. Eckardt scores pre- and pos-retreatment in these three patients were 7 and 3, 4 and 2, and 3 and 2 , respectively. The patient who did not respond to repeated POEM had treatment naïve type II Achalasia (before the index POEM), with a non-sigmoid esophagus. The Eckardt score was 5 before retreatment and remained the same after retreatment.

When assessing symptomatic reflux, one-third of the cohort $(32.1 \%)$ reported reflux symptoms, of which $88 \%$ were on PPI. Regarding use of PPI, around half of the patients (53.7\%) were 


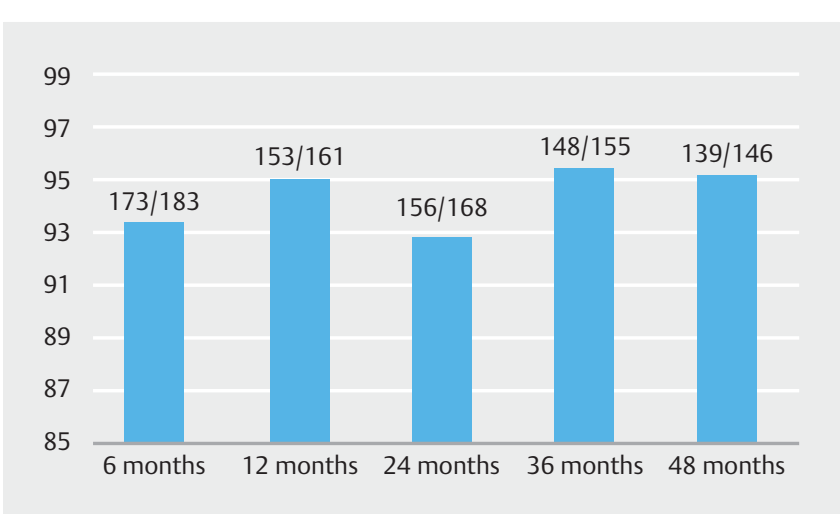

- Fig. 1 Clinical success (Eckardt score $\leq 3$ ) at different time intervals after POEM in patients who had follow-up at 48 months.

not taking PPI at 48 months post-POEM, $35.3 \%$ were on daily $\mathrm{PPI}$, and $11 \%$ were on PPI as-neeed. Post-procedure upper endoscopy was performed in 131 patients: $16.8 \%(22 / 131)$ had evidence of reflux esophagitis (Grades $A=13, B=6, C=1$, $D=2$ based on the LA classification). Of these 22 patients, 19 (86.4\%) were on daily PPI while $3(13.6 \%)$ were taking PPIs occasionally. Post-POEM pH monitoring was performed in $80 \mathrm{pa}-$ tients: $38(47.5 \%)$ had esophageal acid exposure time $>6 \%$ and $44(55 \%)$ had a DeMeester score $>14.72$ ( $>$ Table 2$)$. There was no difference in the incidence of symptomatic reflux between patients who underwent posterior vs anterior approach POEM (OR 1.34, 95\% Cl: 0.59-3.07; $P=0.480$ ).

A total of eight AEs occurred in eight patients (AE rate: $5.5 \%$, Cl: 2.4-10.5). AEs included: 6 mucosotomies and 2 pneumothoraxes (Graded as mild $(n=6)$ and moderate $(n=2)$ as per the ASGE Lexicon classification). All mucosotomies were recognized during the procedure and were successfully closed using standard endoscopic clips or over the scope clips (OTSC). There were no extra-esophageal leaks identified at the mucosotomy sites on post-operative esophagram. Both patients with pneumothorax were successfully managed with a chest tube.

\section{Discussion}

This study has a number of important findings when it comes to long-term outcomes of POEM. Firstly, POEM has a high initial clinical success rate durable over time (clinical success over $95 \%$ at 4 years). Secondly, AEs were uncommon with no longterm morbidity. Our findings on the early clinical success and AEs of POEM are in keeping with previously published studies that report findings after a shorter follow-up time. Inoue et al [5], reported a short-term clinical success of $91.3 \%$ at 2 months in 500 patients in a single-center series from Japan. Li et al [14] validated this finding in a single center Chinese study on 562 patients with a reported clinical success rate of $94 \%$ within the first year. Both series reported a longer follow-up response on a limited number of patients. Inoue et al reported a durable clinical success of $88.5 \%$ at 3 years in 61 patients whereas Li et al reported a drop in the long-term durability of the early clinical success of POEM in only 48 of 562 patients, with $87 \%$ having a
- Table 2 Procedure characteristics and outcomes.

\begin{tabular}{|c|c|c|}
\hline & $\begin{array}{l}\text { Number of } \\
\text { patients with } \\
\text { available data } \\
\mathrm{N}=14 \text { if not } \\
\text { specified }\end{array}$ & $\begin{array}{l}\text { Overall } \\
\text { N (\%) }\end{array}$ \\
\hline $\begin{array}{l}\text { Full thickness LES myotomy; } \\
\text { (n) }(\%)\end{array}$ & 145 & $76(52.4)$ \\
\hline $\begin{array}{l}\text { Gastric myotomy }(\mathrm{cm}) ; \\
(\text { mean } \pm \mathrm{SD})\end{array}$ & & $3.2 \pm 1$ \\
\hline $\begin{array}{l}\text { Esophageal myotomy }(\mathrm{cm}) \text {; } \\
(\text { mean } \pm \mathrm{SD})\end{array}$ & & $8.5 \pm 2.5$ \\
\hline $\begin{array}{l}\text { Orientation of myotomy } \\
\text { (anterior); (n) (\%) }\end{array}$ & 144 & $112(77.8)$ \\
\hline $\begin{array}{l}\text { Patients with adverse } \\
\text { events; }(n)(\%)\end{array}$ & & $8(5.5)$ \\
\hline
\end{tabular}

Follow up-post poem

\begin{tabular}{|c|c|c|}
\hline $\begin{array}{l}48 \text { months Eckardt; (Median) } \\
(\text { IQR)) }\end{array}$ & & $1(0-2)$ \\
\hline $\begin{array}{l}36 \text { months Eckardt; (Median) } \\
\text { (IQR) }\end{array}$ & 123 & $1(0-2)$ \\
\hline $\begin{array}{l}24 \text { months Eckardt; (Median) } \\
(\text { IQR)) }\end{array}$ & 135 & $1(0-2)$ \\
\hline $\begin{array}{l}12 \text { months Eckardt; (Median) } \\
\text { (IQR) }\end{array}$ & 123 & $1(0-2)$ \\
\hline $\begin{array}{l}6 \text { months Eckardt; (Median) } \\
\text { (IQR) }\end{array}$ & 141 & $1(0-1)$ \\
\hline $\begin{array}{l}\text { HRM Resting pressure } \\
(\mathrm{mmHg}) ;(\text { mean } \pm \mathrm{SD})\end{array}$ & 93 & $16.9 \pm 9$ \\
\hline $\begin{array}{l}\text { HRM IRP }(\mathrm{mmHg}) ; \text { (mean } \pm \\
\text { SD) }\end{array}$ & 89 & $10.28 \pm 7.1$ \\
\hline Symptomatic reflux; (n) (\%) & 140 & $45(32.1)$ \\
\hline Esophagitis; (n) (\%) & 131 & $22(16.8)$ \\
\hline $\begin{array}{l}\text { Abnormal acid exposure; } \\
\text { (n) (\%) }\end{array}$ & 80 & $38(47.5)$ \\
\hline DeMeester >14.72; (n) (\%) & 80 & $44(55)$ \\
\hline PPI use; (n) (\%) & 136 & \\
\hline Yes-daily & & $48(35.3)$ \\
\hline Yes-occasionally & & $15(11)$ \\
\hline No & & $73(53.7)$ \\
\hline $\begin{array}{l}\text { Follow up time (months); } \\
\text { (Median)(IQR) }\end{array}$ & & $55(49.9-60.6)$ \\
\hline \multicolumn{3}{|c|}{$\begin{array}{l}\text { HREM, high-resolution esophageal manometry; IRP, integrated relaxation } \\
\text { pressure; LES, lower esophageal sphincter; IQR, interquartile range; PPI, } \\
\text { proton pump inhibitor }\end{array}$} \\
\hline
\end{tabular}

clinical response at $\geq 4$ years. The tendency toward decreased efficacy in the long-term following POEM $[5,9,12,18-22]$ is also apparent in LHM [23]. The study published in 2018 by Teitelbaum et al also showed a decrease in the clinical success rate 
of POEM in Achalasia patients to around $83 \%$ at 5 years followup. This has been traditionally explained by the degenerative nature of Achalasia and the fact that all available treatments are rather palliative than curative [24]. On the other hand, Hungness et al reported the durability of POEM after following 115 patients for an average of 2.4 years post-POEM, showing a stable clinical success rate of $92 \%$. With a 4 -year clinical response rate of $95.2 \%$, our study showed long-term results that were not matched in previous publications. Despite the fact that our outcome was retrospectively recorded in the majority of our cohort, it is important to note that published studies with reported lower success rates had relatively small sample size, with a maximum of 61 patients reported by Inouel et al [5]. In a recently RCT published by our group [25], there was a reported higher clinical efficacy at 1-year post procedure.

One argument that can be made against the validity of our results is survivorship bias. One can argue that patients who were lost to follow-up before the 4-year time point might have had clinical recurrence and thus our results underestimate the true clinical response rate at this time point. We have included - Fig. 1 showing the clinical response rate of all the patients at different time points leading to the 4-year end point. By presenting this data, we are showing that there is no trend in which the attrition of our patients was systematically linked to the recurrence of symptoms. Moreover, it is also important to note that the number of patients who were lost to follow-up due to recurrence could be offset by the number of patients who were lost to follow-up due to not having any symptoms (Achalasia or GERD) and thus had no motivating reason to follow-up.

Interestingly, our study also didn't detect any significant difference between the clinical success at 48 months between the different Achalasia subtypes in contrast to previously published results [26] that reported a lower efficacy of POEM in Achalasia subtype III when compared to other subtypes. This may probably be due to our study being underpowered to detect a true difference, since there were only nine patients with type III Achalasia. In fact, to detect our observed difference in clinical response between subtypes I (97.6\%) and III (88.9\%) as a true significant difference, we would need 127 patients in each group.

The studies by Inoue [5] and Li [12] et al reported that procedure-related AEs were uncommon, occurring in 3.2\% and $6.4 \%$ of patients, respectively, and comparable to an adverse event incidence of $5.5 \%$. In the current study, serious AEs requiring surgery were not reported. Moreover, the need for surgical management of a complication from POEM has been reported to be $0.2 \%$ in a meta-analysis of over 551 POEMs [27].

The current study also reported on reflux symptomatology $\geq 48$ months post-POEM. Reflux rates were significant, with one third of patients reporting symptoms and over half of patients taking PPI either daily or as needed, at least 4 years after POEM. The reflux rate reported in this study is lower than that reported in the literature [28]. This difference could be explained by the longer follow-up duration of our study. There is a possibility that post-POEM reflux symptoms could be transient post-procedure. Also, it is important to note that, depending on the study center, some patients were started on prophylactic PPI as part of the center's post-POEM follow-up protocol. In fact, we have 25 patients in our cohort who did not reported any symptomatic post-POEM reflux and were taking PPI either daily (18) or as-needed (7). These patients may have had clinically significant reflux that was adequately treated with PPI. In regards to the objective evaluation of reflux, a recent meta-analysis reported abnormal esophageal acid exposure in $39 \%$ and $16.8 \%$ of patients following POEM and LHM, respectively [29]. Reflux esophagitis was also significantly higher following POEM as compared to LHM (29.4\% vs $7.6 \%)$. Published results of abnormal acid exposure time and esophagitis following POEM are similar to the results from the current study. In addition, more than half the patients in this study required PPI for acid suppression. Regarding long-term management of postmyotomy reflux, the ability to perform a simultaneous fundoplication at the time of LHM has been an advantage of this approach over POEM [30] However, the introduction of transoral fundoplication to the arsenal of tools used to manage reflux is putting that advantage to test. It has been shown by Tyberg et al [31], on a small cohort of patients that TIF after POEM is a feasible, safe, and efficacious technique for management of postPOEM reflux. There are other ongoing efforts [32,33] to report the efficacy and safety of transoral fundoplication on larger cohorts of post-POEM patients with reflux. Moreover, we recently published a case report [34] which also showed that POEM-TIF in the same session is an innovative and feasible approach to manage post-POEM reflux symptoms. However, it remains a challenge to select the patients who would benefit most from an anti-reflux procedure whether it is in the same session or even post-POEM. Thus, we need more data on the efficacy of the mentioned anti-reflux techniques in the POEM population.

Our study shows that POEM has favorable long-term outcomes as well as an impressive perioperative safety profile and acceptable long-term complication rates. It is the largest study to report long-term clinical outcomes after POEM. In addition to its retrospective nature, there are several other limitations of this study. It is important to note that while the Eckardt score is a simple, standardized, and commonly used symptom score to assess Achalasia outcomes, its use as the primary method to evaluate clinical response to therapy can be limited by its subjective nature and potential interviewer variability. However, the Eckardt score is still considered a universal score to assess clinical outcomes of available Achalasia therapies, therefore, its use in our study is justifiable. In addition, as explained earlier, our study was underpowered to detect any true difference in the efficacy of POEM between the different subtypes of Achalasia due to the small number of patients with subtype III. Finally, there was a proportion of patients who did not have long-term data on $\mathrm{pH}$ monitoring and, thus, the reported incidence on abnormal esophageal acid exposure may not be accurate. Given the retrospective nature of the study, symptomatic GER was assessed mainly by chart review instead of standardized methods like the GERD questionnaires. The low incidence of AEs and high success rates seen in the present study may not be generalizible outside tertiary care centers, as procedures were performed by experienced high-volume endoscopists. 


\section{Conclusion}

In conclusion, POEM is a highly effective, safe, and minimally invasive technique to treat Achalasia with excellent long-term efficacy. Attention to post-POEM GER needs to be addressed with standardized diagnostic tools, and newly available antireflux treatment investigated in this special population.

\section{Competing interests}

Vivek Kumbhari is a consultant for Boston Scientific, Apollo Endosurgery, Medtronic, ReShape Medical. Mouen A. Khashab is a consultant and advisory board for Boston Scientific and consultant for Olympus.

\section{References}

[1] Khashab MA, El Zein M, Kumbhari V et al. Comprehensive analysis of efficacy and safety of peroral endoscopic myotomy performed by a gastroenterologist in the endoscopy unit: a single-center experience. Gastrointestinal endoscopy 2016; 83: 117-125

[2] Parsa N, Khashab MA. POEM in the Treatment of esophageal disorders. Curr Treatment Options Gastroenterol 2018: doi:10.1007/ s11938-018-0168-0

[3] Inoue H, Minami H, Kobayashi Y et al. Peroral endoscopic myotomy (POEM) for esophageal achalasia. Endoscopy 2010; 42: 265-271

[4] Von Renteln D, Fuchs KH, Fockens P et al. Peroral endoscopic myotomy for the treatment of achalasia: an international prospective multicenter study. Gastroenterology 2013; 145: 309-311

[5] Inoue $\mathrm{H}$, Sato $\mathrm{H}$, Ikeda $\mathrm{H}$ et al. Per-oral endoscopic myotomy: a series of 500 patients. J Am Coll Surg 2015; 221: 256-264

[6] Swanstrom LL, Kurian A, Dunst CM et al. Long-term outcomes of an endoscopic myotomy for achalasia: the POEM procedure. Annals Surgery 2012; 256: 659-667

[7] Familiari P, Gigante G, Marchese M et al. Peroral endoscopic myotomy for esophageal achalasia: outcomes of the first 100 patients with short-term follow-up. Annals Surg 2016; 263: 82-87

[8] Schlottmann F, Luckett D], Fine J et al. Laparoscopic Heller myotomy versus peroral endoscopic myotomy (POEM) for achalasia: A systematic review and meta-analysis. Annals Surg 2018; 267: 451-460

[9] Werner YB, Costamagna G, Swanstrom LL et al. Clinical response to peroral endoscopic myotomy in patients with idiopathic achalasia at a minimum follow-up of 2 years. Gut 2016; 65: 899-906

[10] Ngamruengphong S, Inoue $\mathrm{H}$, Chiu PW et al. Long-term outcomes of per-oral endoscopic myotomy in patients with achalasia with a minimum follow-up of 2 years: an international multicenter study. Gastrointest Endosc 2017; 85: 927-933 e922

[11] Akintoye E, Kumar N, Obaitan I et al. Peroral endoscopic myotomy: a meta-analysis. Endoscopy 2016; 48: 1059-1068

[12] Li QL, Wu QN, Zhang XC et al. Outcomes of per-oral endoscopic myotomy for treatment of esophageal achalasia with a median followup of 49 months. Gastrointest Endosc 2018; 87: 1405-1412

[13] Teitelbaum EN, Dunst CM, Reavis KM et al. Clinical outcomes five years after POEM for treatment of primary esophageal motility disorders. Surg Endosc 2018; 32: 421-427

[14] Weber CE, Davis CS, Kramer HJ et al. Medium and long-term outcomes after pneumatic dilation or laparoscopic Heller myotomy for achalasia: a meta-analysis. Surg Laparosc Endosc Percutan Tech 2012; 22: $289-296$
[15] Kumbhari V, Familiari P, Bjerregaard NC et al. Gastroesophageal reflux after peroral endoscopic myotomy: a multicenter case-control study. Endoscopy 2017; 49: 634-642

[16] Eckardt VF, Aignherr C, Bernhard G. Predictors of outcome in patients with achalasia treated by pneumatic dilation. Gastroenterology 1992; 103: $1732-1738$

[17] Cotton PB, Eisen GM, Aabakken L et al. A lexicon for endoscopic adverse events: report of an ASGE workshop. Gastrointest Endosc 2010; 71: 446-454

[18] Li H, Peng W, Huang S et al. The 2 years' long-term efficacy and safety of peroral endoscopic myotomy for the treatment of achalasia: a systematic review. In J Cardiothorac Surg 2019; 14: 1

[19] He C, Li M, Lu B et al. Long-term efficacy of peroral endoscopic myotomy for patients with achalasia: outcomes with a median follow-up of 36 months. Dig Dis Sci 2019; 64: 803-810

[20] Zhang X, Modayil RJ, Friedel D et al. Per-oral endoscopic myotomy in patients with or without prior Heller's myotomy: comparing longterm outcomes in a large U.S. single-center cohort (with videos). Gastrointest Endosc 2018; 87: 972-985

[21] Martinek J, Svecova H, Vackova Z et al. Per-oral endoscopic myotomy (POEM): mid-term efficacy and safety. Surg Endosc 2018; 32: 12931302

[22] Werner YB, Hakanson B, Martinek J et al. Endoscopic or surgical myotomy in patients with idiopathic achalasia. N Engl J Med 2019; 381: 2219-2229

[23] Moonen A, Annese V, Belmans A et al. Long-term results of the European achalasia trial: a multicentre randomised controlled trial comparing pneumatic dilation versus laparoscopic Heller myotomy. Gut 2016; 65: 732-739

[24] Ramchandani M, Nageshwar ReddyD, Nabi Z et al. Management of achalasia cardia: expert consensus statements. J Gastroenterol Hepatol 2018: doi:10.1111/jgh.14097

[25] Khashab MA, Sanaei O, Rivory J et al. Peroral endoscopic myotomy: anterior versus posterior approach: a randomized single-blinded clinical trial. Gastrointest Endosc 2019: doi:10.1016/j.gie.2019.07. 034

[26] Kim WH, Cho JY, Ko WJ et al. Comparison of the outcomes of peroral endoscopic myotomy for achalasia according to manometric subtype. Gut Liver 2017: 642-647

[27] Barbieri LA, Hassan C, Rosati R et al. Systematic review and meta-analysis: Efficacy and safety of POEM for achalasia. United European Gastroenterol J 2015; 3: 325-334

[28] Rosch T, Repici A, Boeckxstaens G. Will Reflux Kill POEM? Endoscopy 2017; 49: 625-628

[29] Repici A, Fuccio L, Maselli R et al. GERD after per-oral endoscopic myotomy as compared with Heller's myotomy with fundoplication: a systematic review with meta-analysis. Gastrointest Endosc 2018; 87: 934-943

[30] Richards WO, Torquati A, Holzman MD et al. Heller myotomy versus Heller myotomy with Dor fundoplication for achalasia: a prospective randomized double-blind clinical trial. Ann Surg 2004; 240: 405-412

[31] Tyberg A, Choi A, Gaidhane M et al. Transoral Incisionless fundoplication for reflux after peroral endoscopic myotomy: a crucial addition to our arsenal. Endosc Int Open 2018; 6: E549-552

[32] Inoue H, Ueno A, Shimamura Y et al. Peroral endoscopic myotomy and fundoplication: a novel NOTES procedure. Endoscopy 2019; 51: 161164

[33] Nabi Z, Ramchandani M, Darisetty S et al. Peroral endoscopic myotomy with endoscopic fundoplication in a patient with idiopathic achalasia. Endoscopy 2020; 52: 74-75

[34] Brewer Gutierrez OI, Benias PC, Khashab MA et al. Same-Session PerOral Endoscopic Myotomy Followed by Transoral Incisionless Fundoplication in Achalasia: Are We There Yet? Am J Gastroenterol 2020; 115: 162 